\title{
Integration und Öffentliche Mobilität: Die Rolle der Planung
}

\section{Oliver Schwedes}

\section{Einleitung}

Nachdem der öffentliche Verkehr in den ersten Nachkriegsjahrzehnten im Zuge der wirtschaftlichen Prosperität stetig an Bedeutung zugunsten des privaten Autoverkehrs verloren hatte, wurde er in den 1970er Jahren wiederentdeckt. Allerdings war das wiedererwachte Interesse der Verkehrsplaner*innen am öffentlichen Verkehr mehrheitlich dadurch motiviert, den rasant wachsenden Autoverkehr zu entlasten, um die Stauanfälligkeit zu reduzieren und damit die ,freie Fahrt für freie Bürger` zu gewährleisten. Damals etabliert sich der verkehrspolitische Konsens, dass der öffentliche Verkehr auch zukünftig als Teil der Daseinsvorsorge, ein Mindestmaß an Mobilität für diejenigen erbringen soll, die sich kein Auto leisten können. Die Alimentierung des öffentlichen Verkehrs legitimiert sich verkehrsplanerisch und -politisch seitdem mit dem Transport derjenigen, die alternativlos im öffentlichen Verkehr gefangen sind - die ,Captives'. Der öffentliche Verkehr als ,Armenkutsche'.

Entgegen allen politischen Lippenbekenntnissen, hat der öffentliche Verkehr, gemessen am privaten Autoverkehr, seitdem die gesellschaftliche Bedeutung einer fest eingeplanten und eingepreisten Restgröße. Demnach haben sich alle Beteiligten auf eine Parallelfinanzierung auf unterschiedlichen Ausgangsniveaus verständigt, mit der das Missverhältnis zwischen öffentlichem und privatem Verkehr seit Jahrzehnten mit jedem Finanzhaushalt von neuem festgeschrieben wird. Wenn der private Verkehr etwas erhält, bekommt der öffentliche Verkehr seinen Anteil - nicht mehr, aber auch nicht weniger. Zwischen 1994 und 2018 wurde der öffentliche Verkehr im Rahmen des Regionalisierungsgesetzes mit rund 172 Mrd.

O. Schwedes $(\bowtie)$

Technische Universität Berlin, Berlin, Deutschland

E-Mail: oliver.schwedes@tu-berlin.de 


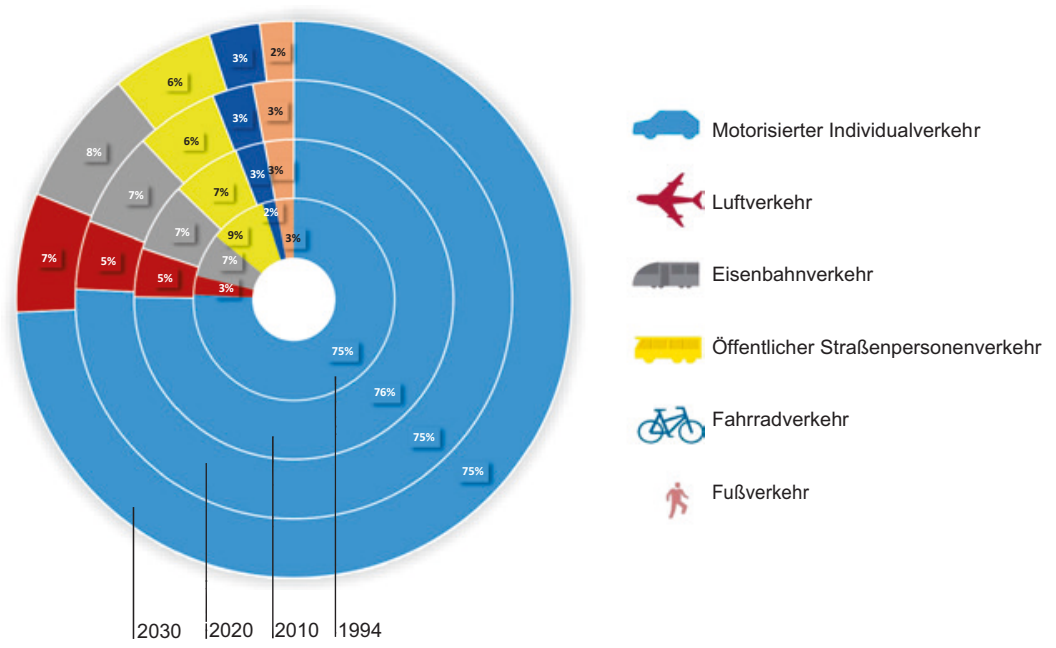

Abb. 1 Anteilige Verkehrsleistung der Verkehrsmittel nach zurückgelegten Personenkilometern. Die Maßeinheit Personenkilometer $(\mathrm{Pkm})$ berechnet sich aus der Anzahl der beförderten Personen multipliziert mit der zurückgelegten Entfernung in Kilometern. (Quelle: Eigene Berechnungen auf Grundlage von Bundesministerium für Verkehr und digitale Infrastruktur (BMVI), Verkehrsverflechtungsprognose 2030, Schussbericht, 11.06.2014, www. bmvi.de/SharedDocs/DE/Anlage/G/verkehrsverfechtungsprognose-2030-schlussbericht-los-3. pdf; BMVI, Verkehr in Zahlen 2019/2020, September 2019, www.bmvi.de/ShareDocs/DE/ Artikel/G/verkehr-in-zahlen_2020.html; eigene Darstellung)

EUR subventioniert. Daraufhin hat sich seine Verkehrsleistung im selben Zeitraum um $36 \%$ erhöht, und die Fahrgastzahlen im öffentlichen Verkehr sind sogar um $56 \%$ gestiegen. Doch parallel zur Subventionierung des öffentlichen Verkehrs erfolgte eine vielfach höhere Förderung des privaten Autoverkehrs, der daraufhin in gleichem Maße gewachsen ist, sodass sich in der Folge des allgemeinen Verkehrswachstums die jeweiligen relativen Anteile am gesamten Verkehrsaufkommen und der Verkehrsleistung nicht verändert haben. Mehr noch, den Prognosen des Bundesministeriums für Verkehr und digitale Infrastruktur zufolge wird sich an diesem Verhältnis auch bis 2030 nichts ändern: Heute wie in der Zukunft entfallen zwei Drittel der Verkehrsleistung im Personenverkehr auf den privaten Autoverkehr (siehe Abb. 1). 
Wenn der öffentliche Verkehr sich zu einer Öffentlichen Mobilität weiterentwickeln soll, deren gesellschaftlicher Stellenwert über eine Residualgröße hinausreicht und einen maßgeblichen Beitrag zu einer nachhaltigen Verkehrsentwicklung leistet, erfordert dies einen entsprechenden verkehrspolitischen und -planerischen Gestaltungswillen. Die aktuelle Anpassungsplanung, die sich heute noch darauf beschränkt, den Status quo zu verwalten, muss von einer Integrierten Verkehrsplanung abgelöst werden. Eine Integrierte Verkehrsplanung zeichnet sich dadurch aus, dass sie normativ, politisch, fachlich und räumlich abgestimmt ist und die verschiedenen Akteure aus den Bereichen gleichberechtigt in die Planung mit einbezieht. Im Folgenden wird gezeigt, wie eine solche Integration in allen vier Bereichen konzipiert sein muss, um diesem Anspruch gerecht zu werden.

\section{$1 \quad$ Normative Integration}

Am Anfang steht mit der normativen Integration die explizite Einbeziehung politischer Werte, an denen sich die Planung ausrichtet. Indem sie sich an gesellschaftlich ausgehandelten Werten orientiert, ist Integrierte Verkehrsplanung ausdrücklich politische Planung. Demgegenüber begreift die herkömmliche Verkehrsplanungszunft, die sich traditionell aus dem Bauingenieurwesen und der Wirtschaftswissenschaft zusammensetzt, Planung als einen wertfreien, naturwissenschaftlich zu begründenden, technischen Prozess. Ihre Vertreter*innen übersehen dabei systematisch die ihrem Handeln zugrunde liegenden impliziten Werturteile. Das zeigt sich anhand des primär verfolgten Ziels, den Verkehr effizienter zu gestalten und den Verkehrsfluss aufrecht zu erhalten, wobei die große Bedeutung des Verkehrs für die Wirtschaftsentwicklung betont wird. Dementsprechend ist der aktuelle Bundesverkehrswegeplan darauf gerichtet, die wachsenden Verkehrsmengen möglichst schnell von A nach B zu leiten (Heuser und Reh 2016). Die Effizienz des Verkehrssystems bemisst sich daran, wie kostengünstig und im Ergebnis wirkungsvoll das Ziel erreicht wird, immer mehr Verkehr mit immer größeren Geschwindigkeiten über immer größere Distanzen zu organisieren. Allerdings kollidiert dieses jahrzehntelang gültige Ziel der ökonomischen Effizienzsteigerung mittlerweile mit den neuen gesellschaftlichen und international ratifizierten Nachhaltigkeitszielen (Vereinte Nationen 2019).

Hier zeigt sich, dass dem vermeintlich wertfreien, naturwissenschaftlich begründeten Planungsziel der ökonomischen Effizienzsteigerung, das die traditionelle Verkehrsplanung versucht durch technische Innovationen zu erreichen (Verkehrsinformationssysteme, neue Antriebstechnologien, autonomes 
Fahren etc.), eine normative Wertentscheidung zugunsten der Wirtschaft und auf Kosten sozialer und ökologischer Werte zugrunde liegt. Ist diese normative Entscheidung einmal gefallen, kann das Verkehrssystem in diesem Sinne von der Verkehrsplanung hochgradig effizient gestaltet bzw. den aktuellen Erfordernissen entsprechend angepasst werden. Die politische Entscheidung fällt aber vorher, wenn es um die Frage geht, ob das Ziel der ökonomischen Effizienzsteigerung auch effektiv ist. Der Ökonom Peter Drucker (1963) hat den Unterschied zwischen Effizienz und Effektivität prägnant so definiert, dass es im ersten Fall darum geht, die Dinge richtig zu tun, während im zweiten Fall angestrebt wird, die richtigen Dinge zu tun. Um die Relevanz der analytischen Unterscheidung zu verdeutlichen, stellte Drucker fest, dass es wohl kaum sinnvoll sei, auf besonders effiziente Weise Ziele zu verfolgen, die man besser gar nicht verfolgen sollte.

Die Vertreter*innen der traditionellen Verkehrsplanung können mit gutem Gewissen sagen, dass sie seit Jahrzehnten eine zweifellos effiziente Anpassungsplanung betreiben, die das Verkehrssystem den ständig wachsenden Verkehrsmengen entsprechend ausbaut und damit den Verkehrsfluss aufrechterhält - sie tun Dinge offensichtlich richtig. Sie verweigern sich aber der politischen Frage, ob das was sie so effizient betreiben auch effektiv ist - tun sie die richtigen Dinge? Aus dieser Einsicht folgt, dass man sich zunächst darüber verständigen sollte, was die richtigen Ziele sind. Das macht die Integrierte Verkehrsplanung indem sie ihre Planungsziele ausdrücklich normativ begründet, transparent vermittelt und öffentlich zur Diskussion stellt.

Im Detail bedeutet dies, dass alle Maßnahmen, welche im Rahmen einer normativ integrierten Planung beschlossen und umgesetzt werden, transparent mit dem Leitbild in Verbindung gebracht werden. So muss sowohl für die Politik als auch für die Zivilgesellschaft nachvollziehbar sein, welche Zielkriterien von der Planung erreicht werden sollen (bspw. Klimaneutralität 2050), welche Strategien hierfür zum Einsatz kommen (bspw. $\mathrm{CO}_{2}$-Reduktion im Verkehrsbereich) und schließlich welche konkreten Maßnahmen hierfür umgesetzt werden (bspw. $\mathrm{CO}_{2}$-Steuer). Eine normative Integration der Planung schafft somit insbesondere Transparenz sowie gesellschaftliche Akzeptanz und ermöglicht es im Folgenden überhaupt erst politische und zivilgesellschaftliche Akteure in die Planung partizipativ vollwertig mit einzubeziehen.

Die gesellschaftliche Etablierung Öffentlicher Mobilität ist auf eine politisch legitimierte Integrierte Verkehrsplanung angewiesen, die die Gestaltung von Mobilität als eine öffentliche Aufgabe begreift, die nicht dem privaten Kalkül jedes Einzelnen überlassen bleiben kann. Das erfordert eine normative Begründung Öffentlicher Mobilität, die glaubhaft vermittelt, dass damit das 
politische Ziel einer nachhaltigeren Verkehrsentwicklung erreicht werden kann. Ein weiteres Qualitätskriterium gelungener normativer Integration, neben der genannten Zielorientierung und der transparenten Prozessgestaltung, ist daher ein evidenzbasiertes Vorgehen. In dem Maße wie auf dem Weg zu einer nachhaltigen Verkehrsentwicklung eine große Transformation des Verkehrssystems erfolgt, geraten schon heute die alten Gewissheiten einer am Auto orientierten Verkehrsplanung in eine Legitimationskrise. Eine Öffentliche Mobilität wird sich nur dann gesellschaftlich legitimieren lassen, wenn gezeigt werden kann, dass sie einen Beitrag zu einer nachhaltigen Verkehrsentwicklung leistet. Die Integrierte Verkehrsplanung hat die Aufgabe, diesen Nachweis wissenschaftlich fundiert zu erbringen. Analog zu der evidenzbasierten Medizin, die sich in den 1990er Jahren durchgesetzt hat, steht die Integrierte Verkehrsplanung für eine evidenzbasierte Mobilitätsplanung.

\section{Politische Integration}

Die zweite Handlungsebene der Integrierten Verkehrsplanung ist die politische Integration. Hier besteht das Ziel darin, die Akteure aus Politik, Verwaltung und Zivilgesellschaft systematisch und dauerhaft miteinander ins Gespräch zu bringen und über alle politischen Ebenen in die Planungs- und Entscheidungsprozesse einzubeziehen. Dieser ambitionierte Ansatz resultiert aus der Erfahrung wachsender Konflikte aufgrund von Sprachlosigkeit zwischen den drei Akteursgruppen. Die Integrierte Verkehrsplanung geht davon aus, dass die Transformation des Verkehrssektors von einer fossilen zu einer postfossilen Mobilitätskultur mit Herausforderungen verbunden ist, die alle drei Akteursgruppen berühren. Die Konfliktlinien verlaufen dabei zwischen Politik und Verwaltung, Verwaltung und Zivilgesellschaft sowie Zivilgesellschaft und Politik. Sie verweisen auf Schnittstellenprobleme, die theoretisch seit langem bekannt sind, praktisch aber bisher nicht hinreichend gelöst werden konnten (Mayntz und Scharpf 1973). Damit Verkehrspolitik und -planung zukünftig handlungsfähig bleiben können, ist heute, anders als in der Vergangenheit, eine stärkere politische Integration zwischen den drei Akteursgruppen notwendig. Welche konkreten Aufgaben sich dabei ergeben, wird im Folgenden anhand der Etablierung einer Öffentlichen Mobilität gezeigt und in Abgrenzung zum aktuellen öffentlichen Verkehr skizziert.

Im Zuge der neoliberalen Entwicklung der letzten dreißig Jahre hat die Politik in Deutschland ihren Gestaltungsanspruch im öffentlichen Verkehr reduziert bzw. an öffentliche Verkehrsunternehmen abgegeben (Engartner 2008; Planka. 
nu 2015). Das hatte direkten Einfluss auf das Verhältnis von Politik und Verwaltung, die zuvor in enger Abstimmung Einfluss auf die damaligen öffentlichen Verkehrsbetriebe genommen hatten. Seit den 1990er Jahren erwartete die Politik sowohl von den in Unternehmen umgewandelten Verkehrsbetrieben wie auch von der Verwaltung zunehmend eine betriebswirtschaftliche Orientierung, deren Maßstab die sogenannte Eigenwirtschaftlichkeit ist. ${ }^{1}$ Das Ziel bestand darin, durch betriebsinterne Reformen die Einnahmen und die Ausgaben in ein ausgewogeneres Verhältnis zu bringen und auf diese Weise die teilweise sehr hohen öffentlichen Subventionen im Verkehr zu reduzieren (Butterwegge et al. 2017). Neben dem ökonomischen Einsparpotential erhoffte sich die Politik von der Marktdynamik die Freisetzung von Innovationsimpulsen, die sowohl zu einer Verwaltungsreform wie auch einer Reform der öffentlichen Verkehrsunternehmen führen sollten. In der Erwartung, dass der über den Markt vermittelte Wettbewerb um die beste Lösung entsprechende Reformen initiieren würde, beschränkte sich die Politik darauf die öffentlichen Finanzmittel in der Verwaltung und den verwaltungsnahen Verkehrsunternehmen stetig zu kürzen. Die Verwaltung und die öffentlichen Verkehrsunternehmen wurden von der Politik weitgehend allein gelassen - das Ergebnis ist eine Entfremdung zwischen Politik und Verwaltung sowie den verwaltungsnahen öffentlichen Verkehrsunternehmen.

Von den öffentlichen Verkehrsunternehmen wurde in dieser Situation erwartet, dass sie einerseits betriebswirtschaftlich agieren andererseits als öffentliche Unternehmen aber auch sozialpolitische Anforderungen erfüllen. Ohne politische Unterstützung war dieser Spagat auf Dauer nicht aufrechtzuerhalten, es hat die Verkehrsunternehmen zerrissen. Damit die Integrierte Verkehrsplanung die angestrebte Öffentliche Mobilität mit dem eingangs formulierten normativen Gestaltungsanspruch im Sinne einer am Gemeinwohl orientierten nachhaltigen Verkehrsentwicklungsstrategie umsetzen kann, muss in einem ersten Schritt die Kommunikation zwischen Politik und Verwaltung wiederaufgenommen werden. Dabei besteht die zentrale Aufgabe darin, das verloren gegangene Vertrauen als Folge der jahrzehntelangen Austeritätspolitik gegenüber einer als ineffizient diskreditierten Verwaltung wiederherzustellen.

Die zweite Konfliktlinie verläuft zwischen der Verwaltung und der Zivilgesellschaft. Hier resultiert die Spannung aus zwei gegenläufigen Motivlagen:

\footnotetext{
${ }^{1}$ Für den öffentlichen Verkehr ist dies in $\S 8$ Abs. 4 im PersonenBeförderungsGesetz (PBefG) festgelegt, ohne das dort definiert wird, was unter Eigenwirtschaftlichkeit zu verstehen ist.
} 
Während die Verwaltung ihre Aufgabe darin sieht, als unbestechliches Werkzeug einer legitimen Regierung zu fungieren, stellt die Zivilgesellschaft demgegenüber gerade diese für sie oftmals rätselhaften administrativen Verfahren infrage und fordert ein Mitspracherecht. Dabei zeichnet sich die deutsche Verwaltung durch eine historisch bedingte, im europäischen Ländervergleich besonders ausgeprägte Intransparenz aus (Seibel 2016, S. 173 ff.). In Verbindung mit der wachsenden Forderung nach Partizipation vonseiten der Bürgerinnen und Bürger, die von der Verwaltung wiederum durch eine verschärfte Blockadehaltung beantwortet wird, hat sich in Deutschland zwischen den beiden ein besonders aufgeladenes Spannungsverhältnis aufgebaut.

Die verfahrene Situation resultiert aus einem wechselseitigen Missverständnis: einerseits verkennt die Verwaltung, dass sie zunehmend auf die Hinweise aus der Zivilgesellschaft angewiesen ist, um dem eigenen Anspruch gerecht zu werden, dem Gemeinwohl zu dienen. Anderseits ereifern sich die Aktivist*innen oftmals dermaßen, dass sie übersehen, wie sehr sie selbst auf eine funktionierende Administration angewiesen sind, wenn die eignen Ziele auf Dauer gestellt werden sollen. Vor diesem Hintergrund setzt die Entwicklung einer Öffentlichen Mobilität eine politische Integration voraus, die beide Seiten zusammenführt. Eine dem Gemeinwohl verpflichtete Öffentliche Mobilität muss heute mehr denn je die vielfältigen Anforderungen in der Bevölkerung berücksichtigen. Dabei ist sie auf die Unterstützung der Verwaltung angewiesen. Das Beispiel der Öffentlichen Mobilität zeigt, dass die politische Integration zwischen Verwaltung und Zivilgesellschaft die Partizipation der Bürgerinnen und Bürgern ermöglichen muss, ohne die Verwaltung zu überfordern. Das heißt, es müssen neue Kanäle geschaffen werden, über die sich die Zivilgesellschaft zu Wort melden kann, und es müssen neue administrative Verfahren entwickelt werden, mit denen es der Verwaltung möglich ist diese zu verarbeiten.

Die dritte Konfliktlinie schließlich verläuft zwischen der Zivilgesellschaft und der Politik (Fischer et al. 2015). Während es im Verhältnis von Verwaltung und Zivilgesellschaft darum geht, einen transparenten Informationsaustausch herzustellen, ist die politische Integration zwischen der Zivilgesellschaft und der Politik auf die aktive Einbindung der Bürger*innen in politische Entscheidungsprozesse gerichtet. Den Hintergrund bildet das wachsende Misstrauen in der Bevölkerung, die sich politisch nicht mehr hinreichend repräsentiert sieht und immer umfangreichere Formen bürgerschaftlichen Engagements entfaltet (Rosanvallon 2017). Die politische Integration verfolgt hier das ambitionierte Ziel, das Menschenrecht gesellschaftlicher Teilhabe für alle Bürger*innen im 
Sinne des umfassenden konzeptionellen Ansatzes der Inklusion zu gewährleisten. ${ }^{2}$ Dabei beschränkt sich der Anspruch der Inklusion nicht darauf, benachteiligte Bevölkerungsgruppen so einzubeziehen, dass ein friedliches Nebeneinander möglich ist; vielmehr besteht das weitreichende Ziel darin, die Gesellschaft so zu gestalten, dass sie den vielfältigen Ansprüchen unterschiedlichster Gesellschaftsgruppen gerecht wird und ein Miteinander ermöglicht.

Dem Anspruch gesellschaftlicher Inklusion folgend konzipiert die Integrierte Verkehrsplanung eine Öffentliche Mobilität für alle Menschen. Anders als der öffentliche Verkehr, der als bescheidene Rückfalloption für eine relativ kleine Bevölkerungsgruppe konzipiert und alimentiert wird, die sich den privaten Autoverkehr nicht leisten kann oder möchte, zielt eine Öffentliche Mobilität auf ein inklusives Verkehrssystem. Die Integrierte Verkehrsplanung orientiert sich dabei an dem Ansatz ,Design für Alle ${ }^{63}$ und konzipiert Öffentliche Mobilität von vornherein so barrierefrei, dass sie für alle gleichermaßen nutzbar ist und nicht mit speziellen Hilfsmitteln ausgestattet werden muss, damit auch benachteiligte Bevölkerungsgruppen Zugang erhalten. ${ }^{4}$ Anstatt beispielsweise eine Rampe zu installieren, die im Bedarfsfall von Rollstuhlfahrenden genutzt werden kann, sollten die Zugänge von Anfang an so gestaltet sein, dass jede*r sie zu jeder Zeit selbstverständlich nutzen kann. Beispielsweise ist eine Niederflurbahn für alle Nutzer*innen hilfreich - nicht nur für Rollstuhlfahrer*innen. Der Planungsansatz ,Design für Alle' ist für die Integrierte Verkehrsplanung besonders anschlussfähig, weil er sich insbesondere dadurch auszeichnet, dass die Nutzer*innen beim Entwicklungsprozess aktiv eingebunden werden, um dem übergeordneten Ziel der Inklusion gerecht zu werden (EDAD 2020).

Ohne dies hier ausführen zu können, soll noch darauf hingewiesen werden, dass die drei skizzierten Dimensionen politischer Integration über alle politischen Ebenen hinweg verfolgt werden müssen, also der Bundes-, Landes- und Kommunalpolitik.

\footnotetext{
${ }^{2}$ Das Menschenrecht auf Inklusion ist in der Behindertenrechtskonvention der Vereinten Nationen festgeschrieben und wurde 2009 von Deutschland unterschrieben. https://www. institut-fuer-menschenrechte.de/monitoring-stelle-un-brk/un-behindertenrechtskonvention/ (Zugriff, 14.04.2020).

${ }^{3}$ Pschyrembel Online https://www.pschyrembel.de/Design\%20f\%C3\%BCr\%20Alle/ S039B/doc/.

${ }^{4}$ Im Unterschied zum Konzept der Barrierefreiheit, ist das Design für Alle bisher weder in Deutschland gesetzlich verankert, noch wird es in Europa an einer Hochschule als grundlegende Planungswissenschaft gelehrt.
} 


\section{$3 \quad$ Fachliche Integration}

Bei der fachlichen Integration ist die interne, auf die eigene Disziplin der Verkehrsplanung bezogene Integration und die externe, auf benachbarte Fachdisziplinen bezogene Integration zu unterscheiden. Erstere befasst sich mit den verschiedenen Planungsfeldern innerhalb der Verkehrsplanung. Während die konventionelle Verkehrsplanung ihre Kernaufgaben in den Bereichen Infrastrukturbau und Verkehrsflusssteuerung sieht, führt die Integrierte Verkehrsplanung als drittes Planungsfeld den Menschen und sein Mobilitätsverhalten ein. Anders als bei der konventionellen Verkehrsplanung, die mit dem Ausbau der Verkehrsinfrastruktur und einer intelligenten Verkehrssteuerung die Aufrechterhaltung des Verkehrsflusses betreibt, geht die Integrierte Verkehrsplanung von den Anforderungen und Bedarfen der Menschen aus und ist darauf gerichtet, sie in einem nachhaltigen Mobilitätsverhalten zu unterstützen. Die drei Planungsfelder erfordern jeweils unterschiedliche Fachkompetenzen, wie das Bauingenieurwesen im Bereich des Infrastrukturbaus, die Wirtschaftsinformatik für die Verkehrsflusssteuerung und die Sozialwissenschaft mit Blick auf das menschliche Verhalten. Mit der internen fachlichen Integration verfolgt die Integrierte Verkehrsplanung eine systematische Verknüpfung der unterschiedlichen Fachdisziplinen, die im Ergebnis mehr ist als die Summe der einzelnen Planungsfelder. Vielmehr erfolgt damit eine grundlegende Veränderung der Zielkoordinaten, indem die Integrierte Verkehrsplanung nicht mehr beim Verkehrsfluss ansetzt, sondern vor dem Entstehen von Verkehr die verkehrsrelevanten Entscheidungen in den Blick nimmt und versucht darauf im Sinne einer nachhaltigen Verkehrsentwicklung einzuwirken (Kutter 2005).

Im Gegensatz zur internen berührt die externe Integration das Verhältnis der Verkehrsplanung zu den benachbarten Fachdisziplinen. Wie bei der internen Integration wird auch in diesem Fall davon ausgegangen, dass eine systematische Kooperation der Verkehrsplanung mit anderen Fachdisziplinen, wie beispielsweise der Gesundheits-, Umwelt- oder Sozialplanung, zu einer neuen Planungsqualität insgesamt beiträgt. Der Mehrwert lässt sich anhand der eingangs erwähnten Wachstumsspirale im Verkehrssektor demonstrieren. Das jahrzehntelange Wettrennen zwischen dem öffentlichen Verkehr und dem privaten Auto ging jedes Mal so aus, wie das zwischen Hase und Igel; obwohl der öffentliche Verkehr immer von sich behauptet hat, besser zu sein als sein Ruf, war das Auto immer schon vorher da. Das liegt daran, dass der öffentliche Verkehr sich in einen Wettkampf einlassen musste, den er nicht gewinnen kann, weil das Auto in der nachfolgend beschriebenen Entwicklungsdynamik immer die attraktivere Alternative darstellt. Demnach sollen immer mehr Menschen immer schneller über 
immer größere Distanzen transportiert werden. Diese Anforderung ergibt sich aus den zunehmend dispersen Raumstrukturen, die dazu führen, dass dieselben Wege heute viel länger sind als vor zwanzig Jahren (UBA 2020). Beispielsweise sind erstmals nach dem Zweiten Weltkrieg sechzig Prozent der erwerbstätigen Bevölkerung dazu gezwungen, zum Arbeitsplatz zu pendeln, wobei die Entfernungen immer größer geworden sind - Tendenz weiter steigend. Die Verantwortung für diese Raumentwicklung liegt aber gerade nicht bei der Verkehrsplanung, die dieser Entwicklung gewissermaßen hinterherläuft, sondern bei der Raum- und Siedlungsentwicklung (Kutter 2019). Indem Gewerbestandorte wie auch Wohnstandorte weit gestreut entstehen, sind sie in vielen Fällen aufgrund der fehlenden Bündelungsfähigkeit nicht oder nur mit unverhältnismäßigem Aufwand für den öffentlichen Verkehr erreichbar. Die einzige Alternative ist dann zumeist das private Auto. Eine fachliche Integration der Verkehrsplanung ermöglicht es also, die Entstehung solcher autoabhängigen Strukturen von Beginn an zu vermeiden.

Indem sich der öffentliche Verkehr an diesem Wachstumsparadigma beteiligt, unterstützt er nicht nur eine Entwicklung, die vor allem dem Auto zugutekommt, darüber hinaus befördert er durch die Anbindung disperser Raumstrukturen selbst eine Entwicklung, die alles andere als nachhaltig ist. Demgegenüber ist eine Öffentliche Mobilität darauf angewiesen, dass die Wachstumsspirale ,höher schneller - weiter' zugunsten einer Strategie der Nahmobilität durchbrochen wird, die auf geringe Geschwindigkeiten und kurze Wege gerichtet ist. Das erfordert eine enge Kooperation zwischen der Verkehrs- und der Raumplanung, die bis heute allenfalls rudimentär praktiziert wird, auch weil die erforderlichen Instrumente fehlen (Hartz 2019; Fürst 2010). Denn viele der verkehrsrelevanten Entscheidungen werden von Unternehmen oder privaten Haushalten bereits dann gefällt, wenn sie ihre Standortwahl treffen. Dabei werden die verkehrlichen Effekte allzu selten mitbedacht. Eine fachliche Integration von Verkehrs- und Raumplanung muss das in Zukunft gewährleisten. Das Beispiel einer neuen Öffentlichen Mobilität zeigt, dass die Integrierte Verkehrsplanung einen interdisziplinären Ansatz verfolgen muss. Um das Ziel einer nachhaltigen Verkehrsentwicklung erreichen zu können, muss sie neben der Raumplanung auch stärker als bisher die schon erwähnten Fachdisziplinen der Gesundheits-, Umwelt-, und Sozialplanung einbeziehen (siehe Abb. 2).

Dadurch eröffnet sich für die Integrierte Verkehrsplanung eine multiperspektivische Sicht, die dem Querschnittsthema Verkehr, das alle gesellschaftlichen Teilbereiche mehr oder weniger stark berührt, gerecht wird. Verkehr wird dann nicht mehr sektoral behandelt, sondern als gesamtgesellschaftliches Phänomen betrachtet und in seiner Bedeutung für den gesellschaftlichen Zusammen- 


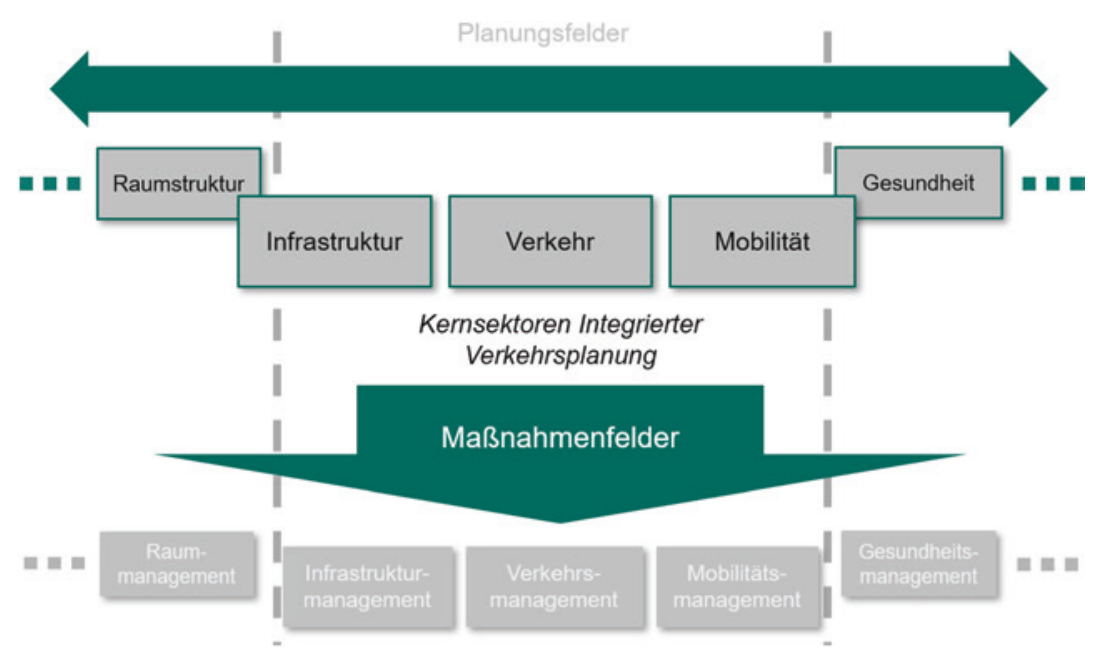

Abb. 2: Fachliche Integration. (Quelle: Schwedes und Rammert 2020)

halt reflektiert. Eine solche multiperspektivische, verschiedene Disziplinen konsultierende Planung erfordert auch neue Formen der Institutionalisierung, die das nicht nur erlauben, sondern auch unterstützen. Das lässt sich negativ am traditionellen öffentlichen Verkehr zeigen, dessen institutionelle Verfassung neue Mobilitätsdienstleistungen nicht erlaubt oder sogar ausdrücklich verhindert. Die Institution des öffentlichen Verkehrs konstituiert sich beispielsweise ganz wesentlich über Gesetzestexte, wie das Personenbeförderungsgesetz (PBefG), in dem klar definiert ist, was zum öffentlichen Verkehr zählt und wie er organisiert sein muss. Demnach muss es sich um liniengebundene Verkehre handeln, die in gewisser Regelmäßigkeit (Verlässlichkeit) in Großgefäßen bestimmte Strecken abfahren und an vorher definierten Haltepunkten die Möglichkeit zum Ein- und Ausstieg geben, bevor sie zum Ausgangspunkt zurückkehren. Damit sind automatisch eine Vielzahl von heute existierenden flexiblen Mobilitätsangeboten ausgeschlossen, die es 1934, als das PBefG formuliert wurde, noch nicht gab. Aber auch die Kultur des öffentlichen Verkehrs ist Teil der institutionellen Verfassung, indem es das Selbstverständnis der Mitarbeiter*innen prägt. Das drückt sich beispielsweise darin aus, dass sich vor der Zentrale der gewaltigen Berliner Verkehrsbetriebe, auf der Länge von 1000 m, kein einziger Fahrradstellplatz befindet, sondern mehrere dutzend Aufkleber an der Fassade angebracht wurden, die das Abstellen des Fahrrads ausdrücklich verbieten. In die Kultur des traditionellen öffentlichen Verkehrs hat das Fahrrad bisher noch keinen Eingang gefunden. 
Demgegenüber verfolgt die Integrierte Verkehrsplanung eine institutionelle Verfassung Öffentlicher Mobilität, die einen Gesetzesauftrag erhält, der den aktuellen gesellschaftlichen Anforderungen entspricht und eine nachhaltige Verkehrsentwicklung verfolgt, die aufgrund ihrer nahräumlichen Orientierung kürzere Distanzen überwinden muss, geringere Geschwindigkeiten erfordert und im Ergebnis weniger Verkehr erzeugt. Die Integrierte Verkehrsplanung folgt dabei den Qualitätskriterien der politischen Integration, indem sie die Ziele öffentlich kommuniziert, die Bevölkerung beteiligt und gemeinsam mit ihr inkludierende Ergebnisse anstrebt. Das Gegenbild zur Öffentliche Mobilität bildet in fast jeder Hinsicht der traditionelle öffentliche Verkehr, der den Wachstumswettbewerb mit dem Motorisierten Individualverkehr weder problematisiert, geschweige denn öffentlich zur Diskussion stellt, die Bevölkerung nicht einbezieht und sich selbst nicht als gesellschaftlich inkludierendes System begreift, sondern darauf beschränkt, die gesellschaftlich Ausgeschlossenen zu bedienen.

\section{Räumliche Integration}

Die räumliche Integration berührt inhaltlich die fachliche Integration im Bereich der oben skizzierten Raumplanung, reicht konzeptionell jedoch darüber hinaus. Mit der räumlichen Integration reagiert die Integrierte Verkehrsplanung auf die Einsicht, dass die Verkehrsentwicklung die administrativen Grenzen auf allen politischen Ebenen - national, regional wie auch kommunal - gesprengt hat. Die Stadtsoziologin Heide Berndt stellte schon Ende der 1960er Jahre in ihrer immer noch lesenswerten Studie über die deutsche Stadtplanungszunft abschließend fest: „Die gegenwärtige Verwaltungseinteilung ist gegenüber den Expansionstendenzen der heutigen Großstädte überholt und verhindert die wirksame Kontrolle über das städtische Wachstum, vor allem eine integrierte Verkehrsplanung [Herv. d. Verf.]. Planungsgemeinschaften zwischen Großstädten und den ihnen anliegenden kleinen Gemeinden sind nur Notbehelfe angesichts einer veralteten Verwaltungsgliederung“ (Berndt 1968, S. 163). Diese Diagnose wurde seitdem immer wieder bestätigt und gilt für die städtische bzw. kommunale Ebene wie auch die regionale und nationale Ebene (Kutter 2019; Priebs 2019). Mit der räumlichen Integration strebt die Integrierte Verkehrsplanung die Kooperation der Akteure unterschiedlicher Gebietskörperschaften über die administrativen Grenzen hinweg an. Damit die Region zur politischen Handlungsebene werden kann, müssen entsprechende Governance-Strukturen aufgebaut werden, in denen „die Akteure vernetzt werden und eine gute Positionierung und Außendarstellung des Gesamtraumes möglich wird“ (Priebs 2019, S. 314; Kiwitt 2019). 
Die Voraussetzung dafür ist, dass die Akteure auf der jeweiligen kommunalen, regionalen oder nationalen Integrationsebene die administrative Grenzen überschreitenden, wechselseitigen Abhängigkeiten erkennen und die Chancen gemeinsamer politischer Gestaltung erfassen. Nur dann kann die Integrierte Verkehrsplanung eine Öffentliche Mobilität etablieren, die langfristig eine nachhaltige Erschließung auf kommunaler, regionaler und nationaler Ebene ermöglicht. Auch hier soll zur Veranschaulichung abschließend der traditionelle öffentliche Verkehr am Beispiel der beiden Bundesländer Berlin und Brandenburg als Kontrastfolien angeführt werden (Bodenschatz 2016). Während die beiden landeseigenen Verkehrsbetriebe (BVG \& ViP) von ihren Landesregierungen politisch stark vertreten werden und entsprechend gestaltungsmächtig sind, gilt dies nicht für den Verkehrsverbund Berlin Brandenburg (VBB), der sich gleichsam zwischen Baum und Borke bewegt. Aus dieser Konstellation resultieren seit über zwanzig Jahren bezüglich des Leitbildes einer Integrierten Verkehrsplanung politische Handlungsblockaden, die sich in einer tiefgreifenden Diskrepanz von programmatischem Anspruch und realer Entwicklung niederschlagen. Im Falle der gemeinsamen Landesplanung Berlin/Brandenburg überlagern sich zwei Konfliktlinien. Erstens zeichnet sich die Zusammenarbeit der beiden Länder seit dem Scheitern der Länderfusion zwar noch durch gemeinsame Interessen, aber immer weniger durch die Gemeinsamkeit der Interessen aus. Vielmehr sind die beiden Länder in einem harten Wettbewerb gegeneinander getreten. Dabei wetteifern sie sowohl um Gewerbeansiedlung wie auch um Wohnbevölkerung, um sich entsprechende Steuereinnahmen zu sichern. Hierbei treten Interessenkonflikte in den Vordergrund, die einer gemeinsamen Handlungsstrategie im Sinne des öffentlich propagierten integrierten Siedlungs- und Verkehrskonzepts entgegenstehen. Zweitens, gewissermaßen quer dazu, konfligieren die Interessen der Landesregierungen mit denen der Kommunen. Dies wirkt sich im Falle Brandenburgs besonders problematisch aus. Selbst dort, wo sich die beiden Länder auf ein gemeinsames Vorgehen einigen, wird dies mit Blick auf die Regionalentwicklung insbesondere durch die Eigeninteressen der brandenburgischen Kommunen konterkariert. Unabhängig von ihrer jeweiligen verkehrlichen Anbindung im Sinne des integrierten Verkehrskonzepts verfügen die Gemeinden über umfangreiche Siedlungsgebiete, die eine weitere Zersiedlung begünstigen (Schwedes 2021, S. 119 ff.). Vor diesem Hintergrund wundert es nicht, dass beide Länder jeweils eigene Verkehrsentwicklungspläne erstellen, anstatt eine räumlich integrierte regionale Strategie zu entwickeln. Unter diesen Bedingungen kann der öffentliche Verkehr keinen Beitrag zu einer nachhaltigen regionalen Verkehrsentwicklung leisten. Aber wie würde eine im Sinne der Integrierten Verkehrsplanung etablierte Öffentliche Mobilität aussehen? 


\section{5 Öffentliche Mobilität}

Bisher wurden die Handlungsfelder der Integrierten Verkehrsplanung erläutert und ihre neue Qualität gegenüber der konventionellen Verkehrsplanung anhand der Öffentlichen Mobilität und in Abgrenzung zum traditionellen öffentlichen Verkehr aufgezeigt. Im Folgenden werden verschiedene Dimensionen Öffentlicher Mobilität beleuchtet, die in der Zusammenschau ein erstes Gesamtbild ergeben. Im Anschluss an diesen Beitrag werden die einzelnen Dimensionen dann in eigenständigen Beiträgen ausgeführt und ein tieferes Verständnis einer Öffentlichen Mobilität vermittelt.

Im Rahmen der Integrierten Verkehrsplanung repräsentiert Öffentliche Mobilität den normativen Anspruch ein nachhaltiges Verkehrsangebot für alle Gesellschaftsmitglieder anzubieten, das gleichermaßen ökonomisch effizient, ökologisch verträglich und sozial gerecht ist (Becker 2019). Dieser grundlegende Anspruch fügt sich in das Konzept der Daseinsvorsorge und entwickelt es zugleich weiter (siehe den Beitrag von Schwedes \& Ringwald in diesem Band). Während der traditionelle öffentliche Verkehr im Rahmen der Daseinsvorsorge die Aufgabe hat, allen Gesellschaftsmitgliedern ein Mindestmaß an Mobilität zur Verfügung zu stellen, bedient er vor allem die soziale Dimension nachhaltiger Verkehrsentwicklung. Um diesen begrenzten Anspruch gerecht zu werden, werden auch hohe finanzielle Subventionen des öffentlichen Verkehrs in Kauf genommen. Demgegenüber formuliert die Öffentliche Mobilität einen deutlich ambitionierteren Anspruch, indem sie neben der sozialen auch die ökologische und die ökonomische Dimension konzeptionell gleichwertig einbezieht. Das bedeutet zum einen, dass die negativen Umweltbelastungen des Verkehrs $\mathrm{zu}$ Buche schlagen und zum anderen die mit dem Verkehr verbundenen ökonomischen Kosten bei der Bewertung Öffentlicher Mobilität einfließen. Demnach kann ein privates Auto schon per Definition nicht Teil der Öffentlichen Mobilität sein, da es im privaten Besitz keinen öffentlichen Zugang erlaubt. Aber auch ein öffentlicher Autobaustein (Carsharing) ist in der Regel das am wenigsten ökonomisch effiziente, ökologisch nachhaltige und sozial gerechte Verkehrsmittel. Für einen Autobaustein im Rahmen Öffentlicher Mobilität müssten die Bedingungen so definiert sein, dass ein hoher Besetzungsgrad garantiert ist, das Fahrzeug möglichst verbrauchsarm und für alle gleichermaßen zugänglich bzw. erschwinglich ist. Diese und andere Bedingungen müssten in einem neuen Personenbeförderungsgesetz zukünftig rechtsverbindlich geregelt sein.

Im Gegensatz zum traditionellen öffentlichen Verkehr, der das Ziel verfolgt, in Konkurrenz mit dem Auto, den Menschen Räume zu erschließen und 
Zugänge zu schaffen, ist die Öffentliche Mobilität darauf gerichtet gesellschaftliche Teilhabe für Alle zu gewährleisten. Ein grundlegendes Missverständnis der konventionellen Verkehrsplanung besteht darin, Raumerschließung und Zugänglichkeit mit gesellschaftlicher Teilhabe gleichzusetzen. Beides kann zusammenfallen, muss es aber nicht und vor allem ist es unter sozialen Nachhaltigkeitsgesichtspunkten nicht wünschenswert (siehe den Beitrag von Daubitz in diesem Band). Vielmehr zielt die Öffentliche Mobilität mit ihrem integrativen Ansatz gerade darauf, gesellschaftliche Teilhabe über Nahbeziehungen zu organisieren und damit Verkehr $\mathrm{zu}$ vermeiden. Dieser positive ökologische Effekt verbindet sich zugleich mit dem sozialen Anspruch die gesellschaftliche Teilhabe für alle zu gewährleisten, die über kurze Wege für alle gleichermaßen barrierefrei gestaltet werden kann. Aus Sicht der Öffentlichen Mobilität stehen sich Ökologie und Soziales daher auch nicht gegenüber, sondern bedingen sich wechselseitig (Brocchi 2019). Indem eine ökologisch verträgliche Mobilität auf Nahbeziehungen gerichtet ist, die allen Gesellschaftsmitgliedern die soziale Teilhabe ermöglichen, ist sie auch sozial gerecht und, indem die gesellschaftlichen Kosten insgesamt gesenkt werden, im Ergebnis auch ökonomisch effizient. Eine so verstandene Öffentliche Mobilität, die als Teil der staatlichen Daseinsvorsorge dem Gemeinwohl dient, lässt sich auf das verfassungsrechtlich garantierte Sozialstaatsprinzip zurückführen und ist anders als der öffentliche Verkehr kein Almosen, sondern ein Grundrecht (Hill 1996).

Als Teil einer neuformulierten Daseinsvorsorge bildet die Öffentliche Mobilität eine institutionelle Verfassung für die Vielzahl neuer Mobilitätsdienstleistungen (siehe den Beitrag von Wolking in diesem Band). Während das ursprüngliche Konzept der Daseinsvorsorge einen autoritären Staat voraussetzte, der die staatlichen Vorsorgeleistungen wie den öffentlichen Verkehr selbst erbrachte, liegt der Öffentlichen Mobilität ein anderes Staatsverständnis zugrunde. Zum einen ist der hierarchisch strukturierte und zentral gesteuerte Staat nicht mehr die einzige politische Instanz, die über die Ausgestaltung Öffentlicher Mobilität entscheidet. Vielmehr sehen sich die staatlichen Instanzen einer wachsenden Zahl zivilgesellschaftlicher Akteure gegenüber, die sich an verkehrspolitischen Entscheidungsprozessen beteiligen und damit Einfluss auf die öffentliche Planung nehmen - der Staat hat Gesellschaft bekommen (Schuppert 2008, S. 189). Zum anderen versteht sich der Staat immer weniger als Leistungsstaat, der die öffentlichen Aufgaben selbst erbringt, sondern begreift sich zunehmend als Gewährleistungsstaat, der die Erfüllung öffentlicher Aufgaben gewährleistet: „Der Staat [...] sieht in immer weiteren Bereichen (vornehmlich der Daseinsvorsorge) davon ab, die ihm obliegenden Aufgaben selbst wahrzunehmen, sondern ermöglicht Privaten im Rahmen hoheitlich gesetzter regulativer 
Vorgaben (z. B. Zielsetzungen, Verfahrensregeln, Organisationsmodelle) die Aufgabenerledigung [...]" (Schoch 2008, S. 241 f.).

Für die Öffentliche Mobilität heißt das, dass sie die privaten Anbieter neuer Mobilitätsdienstleistungen über Organisationsmodelle und Verfahrensregeln einbindet und im Sinne der am Gemeinwohl orientierten Zielsetzung einer nachhaltigen Verkehrsentwicklung politisch steuert (Knauff 2004). Damit werden die alten Verfahren staatlicher Steuerung im öffentlichen Verkehr durch neue Formen der Governance Öffentlicher Mobilität ersetzt. Eine weitere Aufgabe neben der Einbindung privater Unternehmen bei der Gewährleistung Öffentlicher Mobilität ist die aktive Beteiligung der Bürgerinnen und Bürger (siehe den Beitrag von Schneidmesser in diesem Band). Damit die Öffentliche Mobilität ihrem eigenen Anspruch gerecht werden kann, den Menschen mit seinen Anforderungen und Bedarfen zum Ausgangspunkt verkehrsplanerischer Überlegungen zu machen, ist sie auf einen engen Austausch mit den Bürger*innen angewiesen. Im Gegensatz zum öffentlichen Verkehr, der die Nutzer*innen lange Zeit als ,Beförderungsfälle" behandelte und neuerdings auf die Rolle von Kund*innen reduziert, versteht sich die Öffentliche Mobilität als gesellschaftliches Produkt, dass von den Bürgerinnen und Bürgern politisch mitgetragen wird.

In dem Maße wie sich die Bürger*innen mit Öffentlicher Mobilität identifizieren, verändert sich auch ihre Vorstellung davon. Das lässt sich am Wandel vom öffentlichen Verkehr zum privaten Autoverkehr ablesen. Die Vorstellung von öffentlichem Verkehr ist heute dadurch geprägt, dass es sich bei dem Kollektivverkehr um einen Verkehr des neunzehnten Jahrhunderts handelt, der nicht zu individualisierten, modernen Gesellschaften des zwanzigsten Jahrhunderts passt. Der öffentliche Verkehr, so die dominierende Sichtweise nach dem Zweiten Weltkrieg, sei ein historisches Relikt; die Zukunft gehöre dem Automobil und seinem Versprechen nach individueller Freiheit und Selbstverwirklichung - The American Way of Life (Sachs 1990). Mit den realen Infrastrukturen der Autogesellschaft korrespondieren mentale Infrastrukturen, die sowohl die Vorstellungen und Anschauungen der Menschen prägen wie auch ihr Handeln leiten (Welzer 2011). Die in der Bevölkerung prägende Vorstellung vom Kollektivverkehr bemisst sich dementsprechend am privaten Auto und bewirkt bis heute die selektive Wahrnehmung des öffentlichen Verkehrs als defizitär und unzeitgemäß. Mit dem Kulturwissenschaftler Ernest Gellner (1993) lässt sich sagen, dass dieses System von Vorstellungen und Anschauungen die Kultur des öffentlichen Verkehrs bildet. Dementsprechend sind die Voraussetzungen für eine Kultur der Öffentlichen Mobilität positive Vorstellungen und Anschauungen der Menschen von kollektiv organisierten Verkehren. Eine notwendige wenn auch nicht hinreichende Voraussetzung bildet daher ein Verständnis von Öffentlicher Mobilität 
als ein wesentlicher Beitrag zum Gemeinwohl, wodurch sich die Möglichkeit eröffnet, dass sich auch die Wahrnehmung und Bewertung des privaten Autos wandelt. Ein solcher Kulturwandel ist immer an Erwartungshaltungen gekoppelt, die für Menschen einen bindenden Charakter haben und nur schwer aufzulösen sind. Beispielsweise verbindet sich mit der Autokultur die Erwartung, den Anspruch auf einen kostenlosen Stellplatz im öffentlichen Straßenraum zu haben. Diese Erwartungshaltung resultiert aus einem von vielen Privilegien der Autokultur, die in einer öffentlichen Mobilitätskultur nicht aufrechterhalten werden können. Folglich ist der Wandel von der Autokultur zu einer Kultur Öffentlicher Mobilität notwendigerweise mit sozialen Konflikten und gesellschaftlichen Kämpfen verbunden (siehe den Beitrag von Hoor in diesem Band).

Ein Gesetz ist in einem Rechtsstaat nicht alles, aber alles, was sich nicht auf ein Gesetz berufen kann, wird in einem Rechtsstaat kaum wahrgenommen. Das demonstriert der öffentliche Verkehr, der sich die letzten einhundert Jahre in Deutschland nur deshalb behaupten konnte, weil er über das politische Konstrukt der Daseinsvorsorge als notwendige Gemeinwohlleistung legitimiert ist und diese Leistung rechtsverbindlich in den ÖPNV-Gesetzen der Länder festgeschrieben sind. Das Spezielle an dieser Regelung erkennt man dort besonders gut, wo sie nicht existiert: in den Vereinigten Staaten von Amerika (zum folgenden Jones 2010). In den USA wie in Europa, gerieten die zu Beginn des 20. Jahrhunderts noch privat geführten Verkehrsunternehmen mit der zunehmenden Verbreitung des Autos in eine wirtschaftliche Krise. Während man sich in den europäischen Ländern entschied, die Unternehmen zu verstaatlichen und im Sinne des Gemeinwohls mit öffentlichen Geldern zu subventionieren, überließ man die Verkehrsunternehmen in den USA den Marktkräften. Es gab weder ein politisches Interesse, den öffentlichen Verkehr zu retten, geschweige denn ein Gesetz zu seinem Schutz. Das ist der Grund dafür, dass es heute in den USA nahezu keinen öffentlichen Verkehr mehr gibt.

Für die erfolgreiche Etablierung einer Öffentlichen Mobilität bedeutet das, dass sie auf eine entsprechende Gesetzesgrundlage angewiesen ist, die ein rechtsverbindliche Umsetzung garantiert. Dabei kann an das langbewährte Nahverkehrsgesetz des öffentlichen Verkehrs angeknüpft werden, um es dann im Sinne des umfassenden Gedankens Öffentlicher Mobilität weiterzuentwickeln. Diesen Ansatz verfolgt weltweit einmalig das Berliner Mobilitätsgesetz, indem es neben das Nahverkehrsgesetz ein Radverkehrsgesetz, ein Fußverkehrsgesetz, ein Wirtschaftsverkehrsgesetz sowie ein Gesetz für neue Mobilitätsdienstleistungen stellt (siehe den Beitrag von Kirchner in diesem Band). Das sich dort kein Autoverkehrsgesetz findet, bringt schon die programmatische Stoßrichtung des Berliner Mobilitätsgesetzes zum Ausdruck, das ausdrücklich den Umweltverbund 
auf Kosten des Autoverkehrs fördern will. Eine große Herausforderung wird darin bestehen, die bisher nebeneinanderstehenden Gesetzestexte zum gegebenen Zeitpunkt zu einem Gesetz für Öffentliche Mobilität zu integrieren. Wie das Nahverkehrsgesetz des traditionellen öffentlichen Verkehrs, sollte auch das Gesetz für Öffentliche Mobilität einen finanziellen Rechtsanspruch beinhalten. Dieser hat nicht mehr den Charakter eines Almosen, das dem öffentlichen Verkehr von der Gesellschaft gewährt wird, vielmehr bemisst sich der finanzielle Beitrag für Öffentliche Mobilität daran, wieviel der Gesellschaft eine nachhaltigen Verkehrsentwicklung zukünftig wert ist. Dementsprechend erfordert die Öffentliche Mobilität neue Finanzierungskonzepte, die den gesellschaftlichen Beitrag für eine am Gemeinwohl orientierten nachhaltigen Verkehrsentwicklung angemessen widerspiegeln (siehe den Beitrag von Mietzsch in diesem Band).

Damit die Integrierte Verkehrsplanung den Wandel zur Öffentlichen Mobilität aktiv gestalten kann, benötigt sie entsprechende Informationen. Zwar werden in Deutschland regelmäßig große Verkehrserhebungen durchgeführt, allerdings sind sie so stark aggregiert, dass sie auf kommunaler Ebene - also dort, wo Politik und Planung den Menschen am nächste sind - kaum noch eine Aussagekraft haben. Noch problematischer aus Sicht einer Öffentlichen Mobilität ist es, dass diese quantitativen Erhebungen ausschließlich Verkehr zählen, ihn aber als Maß für Mobilität heranziehen. Eine der größten Erhebungen in Deutschland definiert einen Messwert für Mobilität folgendermaßen: „Eine zentrale Kenngröße zur Beschreibung von Mobilität ist der Anteil der Personen, die an einem Tag mindestens einmal aus dem Haus gehen und damit Verkehr erzeugen: die Mobilitätsquote oder auch der Außer-Haus-Anteil“" (Nobis und Kuhnimhof 2018, S. 25). In der anschließenden Analyse der Daten wird dieser Zusammenhang zwischen „Außer-Haus-Anteil“ - also Verkehr - und Mobilität weiter verstärkt, indem zwischen „mobilen“ und „nicht mobilen“ Menschen unterschieden wird (ebd., S. 25 ff., 131).

Demgegenüber unterscheidet die Integrierte Verkehrsplanung begrifflich zwischen dem Verkehr und der Mobilität, wobei sich letztere nicht an den Verkehrsbewegungen bemisst, sondern am Grad gesellschaftlicher Teilhabe. Anders als der physisch erfahr- und objektiv zählbare Verkehr beschreibt die Mobilität subjektive Möglichkeitsräume für Ortsveränderungen (Schwedes et al. 2018). Die analytische Bedeutung der begrifflichen Unterscheidung von Verkehr und Mobilität kann an einem konkreten Beispiel veranschaulicht werden: Eine Person, die sich die hohen Mieten in der Stadt nicht mehr leisten kann und gezwungen ist, täglich einhundert Kilometer zu ihrem Arbeitsplatz zu pendeln, um dort einem Niedriglohnjob nachzugehen, ist verkehrlich viel unterwegs, ohne sich und ihrer Familie mit dem geringen Lohn eine angemessene gesellschaftliche Teilhabe 
ermöglichen zu können. Entgegen dem Alltagsverstand ist diese Person, die viel Verkehr erzeugt, dennoch nicht besonders mobil. Demgegenüber ist eine gut ausgebildete Stadtbürgerin, die ihren Arbeitsplatz fußläufig erreicht und über ein hohes Einkommen verfügt, hochgradig mobil. Denn sie ist in der Lage, das vielfältige Angebot der Stadtgesellschaft in vollen Zügen zu genießen, indem sie sich nicht zuletzt die hohen Mieten leisten kann, allerdings ohne viel Verkehr zu erzeugen.

Die begriffliche Unterscheidung von Verkehr und Mobilität ermöglicht es, gesellschaftliche Teilhabe zu thematisieren, ohne sie notwendig mit Verkehr zu verbinden. Dementsprechend hat sich das Konzept der Öffentlichen Mobilität zum Ziel gesetzt, allen Bürger*innen eine angemessene gesellschaftliche Teilhabe (Mobilität) zu gewährleisten, ohne dass sie dazu auf ein stetig wachsendes Verkehrsaufkommen angewiesen sind, indem sie sich beispielsweise gezwungen sehen, ein Auto anzuschaffen, um jeden Tag zur Arbeit pendeln zu können. Damit die Integrierte Verkehrsplanung die Menschen darin unterstützen kann, unabhängig von wachsenden Verkehrsmengen hinreichend mobil zu sein, benötigt sie, ergänzend zu den Verkehrsdaten, Daten zur Mobilität. Die Öffentliche Mobilität muss sich daran messen lassen, ob sie dem eigenen Anspruch gerecht wird und eine angemessene gesellschaftliche Teilhabe gewährleisten kann. Um dies zukünftig bewerten zu können, benötigt die Integrierte Verkehrsplanung entsprechende Kriterien (siehe den Beitrag von Rammert in diesem Band).

Ebenso wie die Daten dort erhoben werden müssen, wo die Menschen ihren Alltag erleben, bildet die kommunale Ebene auch das wichtigste verkehrspolitische Gestaltungsfeld, weil hier auf die konkreten Anforderungen und Bedarfe der Menschen eingegangen werden kann. Damit die Städte und Gemeinden handlungsfähig bleiben können, müssen sie die verkehrspolitischen Herausforderungen vor Ort kennen und eine Vorstellung davon entwickeln, welche Ziele sie langfristig erreichen wollen. Dazu ist es erforderlich, dass sie sich im dauerhaften Dialog mit ihren Bürger*innen ein angemessenes Verständnis der kommunalen Mobilitätsverhältnisse erschließen. Das erfordert eine kontinuierliche Dokumentation und Evaluation der durchgeführten Maßnahmen und der Ergebnisse, die es ermöglicht, bewährte Eingriffe zu würdigen und aus Fehlern zu lernen. Vor dem Hintergrund der sich abzeichnenden großen Transformation der Stadträume und ihrer damit verbundenen Neuaufteilung, die für alle Beteiligten neue Herausforderungen mit sich bringen, stellt eine sogenannte Mobilitätsberichterstattung ein wichtiges und notwendiges Planungsinstrument dar (siehe den Beitrag von Hausigke und Kruse in diesem Band).

Die große Bedeutung valider Daten für eine informierte politische und planerische Gestaltung hat sich zuletzt auf dramatische Weise in der weltweiten 
Corona-Pandemie gezeigt. Dass Deutschland die Ausbreitung so gut eindämmen konnte, lag vor allem daran, dass es nicht das erste betroffene Land war. Deutschland war damit zum einen vorgewarnt und konnte sich auf die Pandemie vorbereiten, zum anderen hatte es aufgrund der Erfahrungen der schon betroffenen Länder einen Informationsvorsprung, den es nutzen konnte. Dennoch war die Situation aufgrund fehlender Informationen wochenlang unübersichtlich: es war selten bekannt, wer wen wann angesteckt hatte, wie viele Menschen schon infiziert sind und wie sich die Infektion ausbreitet. Die Politik konnte deshalb lange Zeit nur wenig gezielt agieren, zumal sich die Informationslage immer wieder änderte. Eine aktive Gesundheitsplanung war unter diesen Bedingungen kaum möglich; vielmehr waren alle Beteiligten gezwungen, sich der ständig wandelnden Lage immer wieder neu anzupassen. Was in der Gesundheitspolitik eine extreme Ausnahmesituation ist, bildet in der Verkehrspolitik mit der sogenannten Anpassungsplanung bis heute den Normalzustand. Während sich in der Gesundheitspolitik vor dreißig Jahren eine evidenzbasierte Planung etabliert hat, die auf einer gesetzlich vorgegebenen Berichterstattung fußt, fehlt der Verkehrspolitik auch heute noch ein vergleichbares Planungsinstrument.

\section{Fazit}

Den Ausgangspunkt des Beitrags bildete die These: Der öffentliche Verkehr ist tot, es lebe die Öffentliche Mobilität! Eine wesentliche Grundlage für die Transformation des öffentlichen Verkehrs in eine Öffentliche Mobilität bildet eine Integrierte Verkehrsplanung. Ihr liegt die basale verkehrswissenschaftliche Einsicht zugrunde, dass es sich bei Verkehr um ein gesellschaftliches Phänomen handelt, das in all seinen Facetten und Wirkgefügen betrachtet werden muss. Der Verkehr ist sowohl Ausdruck historisch-spezifischer gesellschaftlicher Verhältnisse wie auch ein diese Verhältnisse prägendes Element. Die jeweilige Technik geht mit den sozialen Verhältnissen eine Verbindung ein, die immer schon ganz unterschiedliche Ausprägungen haben konnte - das gilt für die Eisenbahngesellschaft der Vergangenheit ebenso wie für die Autogesellschaft der Gegenwart. Dementsprechend muss sich Verkehrspolitik als Gesellschaftspolitik begreifen und die Integrierte Verkehrsplanung hat den Anspruch gesellschaftliche Verhältnisse zu gestalten, anstatt sie wie die konventionelle Verkehrsplanung bis heute zu verwalten (Schwedes 2018).

Dieser Gestaltungsanspruch Integrierter Verkehrsplanung sieht sich mit dem etablierten großtechnischen System Automobilität konfrontiert, das sich über viele Jahrzehnte überaus erfolgreich bewährt hat und dessen Institutionen dem Wandel zu einer Öffentlichem Mobilität im Weg stehen. Dem Technikhistoriker Jürgen Radkau (1991, S. 8) zufolge gibt es erfahrungsgemäß zwei Zeitfenster, 
in denen eine gesellschaftliche Steuerung technischer Großsysteme möglich erscheint. Zum einen, wenn sie noch in den Kinderschuhen stecken, gerade dann neigen wir aber dazu, unsere Wunschträume hineinzuprojizieren, anstatt die Technik im gesellschaftlichen Kontext kritisch zu reflektieren. Zum anderen im Alter, wenn sie ihren Zenit überschritten haben und ihre gesellschaftliche Prägekraft nachlässt. Dann besteht die Schwierigkeit jedoch darin, zu bestimmen, in welchem Stadium sich ein System gerade befindet.

Hier ist freilich zu ergänzen, dass ein technisches Großsystem eben kein Lebewesen ist und organische Phasenmodelle deshalb nicht weit tragen. So wurde das Ende des Automobils in den letzten vierzig Jahren schon öfters ausgerufen, um sich im Nachhinein ebenfalls als Wunschtraum zu entpuppen. Auch in den letzten zehn Jahren stieg die Zahl der privat gehaltenen Autos in Deutschland von 37 auf 42 Mio. und die Prognosen gehen von weiteren Wachstumsraten aus. Demnach erscheint das großtechnische System Auto sehr vital und keinesfalls am Ende seiner Lebensspanne. Aber wahrscheinlich ist es nicht richtig, auf das vermeintliche Ableben eines technischen Großsystems zu warten, um politisch einzugreifen. Denn die Integrierte Verkehrsplanung will ja gerade auf gesellschaftliche Verhältnisse verändernd einwirken. Ohne diesen Anspruch aufzugeben, sollte sich Planung heute aber zugleich eine realistische Bescheidenheit gegenüber den Möglichkeiten der Steuerung großtechnischer Systeme erhalten.

\begin{abstract}
„Um ein realistisches Bild von der Genese großer Systeme zu erlangen und nicht Planbarkeitsillusionen zu verfallen, ist es wichtig, nicht nur die planmäßigen, sondern auch die ungeplanten Elemente bei der Entstehung von Systemen zu beachten. Weiträumige und komplizierte technische Systeme könnten kaum je entstehen, wenn sie von Anfang an in dieser Form projektiert werden müssten, denn im Allgemeinen fehlen Instanzen mit einem derartigen Planungs- und Implementationsvermögen. Die großen Netzwerke der Stadttechnik entwickelten sich zu einer Zeit, als es noch kaum irgendwo eine institutionalisierte Stadtplanung gab. Aber Vernetzung geschieht auch fortwährend ohne großen Plan: einfach durch die Begrenztheit des verfügbaren Raumes“"(Radkau 1991, S. 7).
\end{abstract}

Womöglich bildet auch heute der begrenzte städtische Raum den Anlass für eine Verkehrswende, die sich gegen das Automobil wendet. Anders als in der Vergangenheit, als die Verkehrsplanung mit Masterplänen auftrat, um die Städte nach ihrem Bild zu formen, besteht die Aufgabe der Integrierten Verkehrsplanung heute nicht darin, den Plan zu liefern. Vielmehr hält sie den gesellschaftlichen Gestaltungsanspruch aufrecht, indem sie alle Beteiligten dazu einlädt, einen Zielkorridor zu definieren, in dem man sich zukünftig gemeinsam bewegen möchte, um das politisch vorgegebene Ziel einer nachhaltigen Verkehrsentwicklung zu 
erreichen. Wenn sich bei diesem gesellschaftlichen Aushandlungsprozess herausstellen sollte, dass das Auto im Weg steht, könnte es früher als erwartet dem neuen großtechnischen System Öffentlicher Mobilität Platz machen. Die Vielzahl verkehrspolitischer Initiativen aus den Reihen der Zivilgesellschaft verweisen ebenso darauf, wie der wachsende politische Legitimationsverlust alter etablierter Akteure im Politikfeld Verkehr, wie dem Bundesverkehrsministerium, der deutschen Automobilindustrie, dem Allgemeinen Deutschen Automobilclub, oder der Forschungsgemeinschaft für das Straßen- und Verkehrswesen, um nur einige wenige zu nennen.

\section{Literatur}

Becker, Udo. 2019. Worin liegt das Ziel aller Verkehrsplanung und wie verträgt sich das mit konsequentem Umweltschutz? In Handbuch der kommunalen Verkehrsplanung (Loseblattsammlung), Hrsg. T. Bracher, K. Dziekan, J. Gies, F. Huber, F. Kiepe, U. Reutter, K. Saary, O. Schwedes, Beitragsnummer 3.1.1.1. Berlin/Offenbach: VDE.

Berndt, Heide. 1968. Das Gesellschaftsbild bei Stadtplanern. Stuttgart: K. Krämer.

Bodenschatz, Harald. 2016. Visionen gesucht - Die Großstadtregion Berlin. Merkur Heft 803: 55-62.

Brocchi, Davide. 2019. Nachhaltigkeit und soziale Ungleichheit. Warum es keine Nachhaltigkeit ohne soziale Gerechtigkeit geben kann. Buchreihe essentials. Wiesbaden: Springer VS.

Butterwegge, Christoph, B. Lösch und R. Ptak. 2017. Kritik des Neoliberalismus. S. 79-122.Wiesbaden: Springer VS.

Drucker, Peter F. 1963. Managing for Business Effectiveness. Harvard Business Review Heft 3, 53-60.

EDAD - Design für Alle - Deutschland e. V. 2020. Barrierefreiheit mit attraktiver Gestaltung verbinden. https://www.design-fuer-alle.de/design-fuer-alle/. Zugriff: 14.04.2020.

Engartner, Tim. 2008. Die Privatisierung der Deutschen Bahn: Über die Implementierung Marktorientierter Verkehrspolitik. Wiesbaden: Springer VS.

Fischer, Frank, A. Durnová, M. Orsini, D. Torgerson, Hrsg. 2015. Handbook of Critical Policy Studies. Cheltenham, UK \& Northampton, MA, USA: Edward Elgar Publishing.

Fürst, Dietrich. 2010. Raumplanung: Herausforderungen des deutschen Institutionensystems. Buchreihe Planungswissenschaftliche Studien zu Raumordnung und Regionalentwicklung, Band 1, Hrsg. H. H. Blotevogel, R. Danielzyk, C. Diller, S. Siedentop, D. Vallée und T. Wiechmann. Lemgo: Dorothea Rohn.

Gellner, Ernest. 1993. Pflug, Schwert und Buch. Grundlinien der Menschheitsgeschichte. Stuttgart: Klett-Cotta.

Hartz, Andrea. 2019. Dynamische Stadtlandschaften. Planerin Heft 3: 14-17. 
Heuser, Tilmann und Werner Reh. 2016. Die Bundesverkehrswegeplanung: Anforderungen an die zukünftige Verkehrsinfratsrukturpolitik des Bundes. In Handbuch Verkehrspolitik, Hrsg. Oliver Schwedes, Weert Canzler, Andreas Knie, 237-264. Wiesbaden: Springer VS.

Hill, Hermann. 1996. In welchen Grenzen ist kommunalwirtschaftliche Betätigung Daseinsvorsorge? Vortrag beim 5. Kommunalpolitischen Forum der IHKs in NRW am 29.8.1996 in Castrop-Rauxel, Manuskript: 8f.

Jones, David W. 2010. Mass Motorization and Mass Transit. An American History and Policy Analysis. Bloomington, IN, USA: Indiana Univ Pr.

Kiwitt, Thomas. 2019. Modell Verband Region Stuttgart. Planerin Heft 3: 21-23.

Knauff, Matthias. 2004. Der Gewährleistungsstaat: Reform der Daseinsvorsorge. Eine rechtswissenschaftliche Untersuchung unter besonderer Berücksichtigung des ÖPNV. Berlin: Duncker \& Humblot.

Kutter, Eckhard. 2019. Stadtstruktur und Erreichbarkeit in der postfossilen Zukunft. Schriftenreihe für Verkehr und Technik, Bd. 99. Berlin: Erich Schmidt.

Kutter, Eckhard. 2005. Entwicklung innovativer Verkehrsstrategien für die mobile Gesellschaft: Aufgaben Maßnahmenspektrum Problemlösungen. Berlin: Erich Schmidt.

Mayntz, Renate, Fritz Scharpf, Hrsg. 1973. Planungsorganisation. Die Diskussion um die Reform von Regierung und Verwaltung des Bundes. München: Piper.

Nobis, Claudia, T. Kuhnimhof. 2018. Mobilität in Deutschland-MiD Ergebnisbericht. Studie von infas, DLR, IVT und infas 360 im Auftrag des Bundesministers für Verkehr und digitale Infrastruktur. Bonn, Berlin.

Planka.nu. 2015. VerkehrsMachtOrdnung. Zur Kritik des Mobilitätsparadigmas. Münster: Unrast.

Priebs, Axel. 2019. Die Stadtregion. Stuttgart: UTB.

Rosanvallon, Pierre. 2017. Die Gegen-Demokratie. Politik im Zeitalter des Misstrauens. Hamburg: Hamburger Edition.

Radkau, Joachim. 1991. Zum ewigen Wachstum verdammt? Historisches über Jugend und Alter großer technischer Systeme. Forschungsgruppe „Große technische Systeme“ des Forschungsschwerpunkts Technik - Arbeit - Umwelt am Wissenschaftszentrum Berlin für Sozialforschung, FS I I 91-505. Berlin. https://bibliothek.wzb.eu/pdf/1991/ii91-505. pdf. Zugriff: 28.04.2020.

Sachs, Wolfgang. 1990. Die Liebe zum Automobil. Ein Rückblick in die Geschichte unserer Wünsche. Hamburg: Reinbek.

Schoch, Friedrich. 2008. Gewährleistungsverwaltung: Stärkung der Privatrechtsgesellschaft? Neue Zeitschrift für Verwaltungsrecht, 241-247.

Schuppert, Gunnar F. 2008. Die neue Verantwortungsteilung zwischen Staat und Gesellschaft - oder: Wessen Wohl ist das Gemeinwohl? vhw Heft 4, 189-193.

Schwedes, Oliver. 2018. Verkehrspolitik als Gesellschaftspolitik. In Verkehrspolitik. Eine interdisziplinäre Einführung, Hrsg. Oliver Schwedes. 3-24. Wiesbaden: Springer VS.

Schwedes, Oliver, S. Daubitz, A. Rammert, B. Sternkopf, M. Hoor. 2018. Kleiner Begriffskanon der Mobilitätsforschung. IVP-Discussion Paper, Heft 1. Berlin.

Schwedes, Oliver. 2021. Verkehr im Kapitalismus. 2. Aufl. Münster: Westfälisches Dampfboot.

Schwedes, Oliver und A. Rammert. 2020. Was ist Integrierte Verkehrsplanung. Eine Selbstverständigung. IVP-Discussion Paper, Heft 2, Berlin. 
Seibel, Wolfgang. 2016. Verwaltung verstehen. Eine theoriegeschichtliche Einführung. Berlin: Suhrkamp.

UBA - Umweltbundesamt. 2020. Mobilität privater Haushalte. https://www.umweltbundesamt.de/daten/private-haushalte-konsum/mobilitaet-privater-haushalte\#verkehrsaufwandim-personentransport (18.06.2020).

Vereinte Nationen. 2019. Ziele für nachhaltige Entwicklung. Bericht 2019. New York. https:// www.un.org/Depts/german/pdf/SDG\%20Bericht\%20aktuell.pdf. Zugriff: 15.04.2020.

Welzer, Harald. 2011. Mentale Infrastrukturen: Wie das Wachstum in die Welt und in die Seelen kam. Schriften zur Ökologie der Heinrich-Böll-Stiftung, Bd. 14.

Open Access Dieses Kapitel wird unter der Creative Commons Namensnennung 4.0 International Lizenz (http://creativecommons.org/licenses/by/4.0/deed.de) veröffentlicht, welche die Nutzung, Vervielfältigung, Bearbeitung, Verbreitung und Wiedergabe in jeglichem Medium und Format erlaubt, sofern Sie den/die ursprünglichen Autor(en) und die Quelle ordnungsgemäß nennen, einen Link zur Creative Commons Lizenz beifügen und angeben, ob Änderungen vorgenommen wurden.

Die in diesem Kapitel enthaltenen Bilder und sonstiges Drittmaterial unterliegen ebenfalls der genannten Creative Commons Lizenz, sofern sich aus der Abbildungslegende nichts anderes ergibt. Sofern das betreffende Material nicht unter der genannten Creative Commons Lizenz steht und die betreffende Handlung nicht nach gesetzlichen Vorschriften erlaubt ist, ist für die oben aufgeführten Weiterverwendungen des Materials die Einwilligung des jeweiligen Rechteinhabers einzuholen.

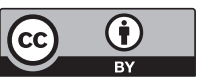

